\title{
Aplicação do Método dos Elementos Finitos na Ele- trostática e Estimulação Magnética Transcraniana
}

Lemos, T. D. A.

Escola Politécnica de Pernambuco

Universidade de Pernambuco

50.720-001 - Recife, Brasil

thiago_domingos15@hotmail.com
Feitosa, M. A. F.

Escola Politécnica de Pernambuco

Universidade de Pernambuco

50.720-001 - Recife, Brasil

marcilio.poli.br

Resumo Este artigo resume os conceitos da estimulação magnética transcraniana, que é uma poderosa técnica não-invasiva de tratamentos e estudos neurológicos. Em combinação com essa técnica, o método dos elementos finitos (MEF) foi adaptado como uma alternativa de diminuir o custo computacional da análise analítica e obter o gradiente do campo elétrico em qualquer ponto no interior da cabeça humana, modelada através de uma esfera homogênea.

\footnotetext{
Abs- $\quad$ This paper summarizes the concepts of transcranial magnetic stimulation, which is a powerful nontract invasive technique for treatments and neurological studies. In combination with this technique, the finite elemento method (FEM) was adapted as an alternative to reduce the computational cost of analytical analysis and obtain the gradiente of the eletric field at any point inside the human head, modeled by a homogeneous sphere.
} 


\section{Introdução}

A estimulação magnética transcraniana (TMS) é uma técnica não-invasiva de estimulação dos tecidos nervosos. Essa técnica vem ganhando aplicação crescente no tratamento da depressão, bem como na área fisioterápica ou de reabilitação muscular. Ademais, uma lista crescente de outras doenças está sob estudo de sua utilização. [1]-[3]

A TMS segue os princípios fundamentais da indução eletromagnética: uma corrente variável no tempo produz um campo magnético variável no tempo e esse campo magnético variável induz um fluxo de corrente elétrica em condutores próximos - incluindo os tecidos humanos. [1][6]

O campo elétrico induzido através da TMS é capaz de despolarizar as membranas celulares das estruturas nervosas através da alteração do potencial transmembrana, e desta maneira gerar um potencial de ação que se propagará em forma de uma onda até atingir células nervosas adjacentes. Nesse processo, células musculares também poderão ser atingidas causando uma contração nas mesmas. [2][3]

Se o potencial intracelular mudar ao longo da direção positiva, então esta é a estimulação excitável ou despolarização, e se o potencial intracelular for alterado ao longo da direção negativa, então essa estimulação é proibitiva ou hiperpolarizante. Se a estimulação na membrana é insuficiente para alcançar o limiar de excitação transmembrana, então a ação potencial na membrana celular não irá ocorrer [2]. Esse limiar é de aproximadamente $-55 \mathrm{mV}$. A Fig. 1 ilustra uma aplicação da TMS.

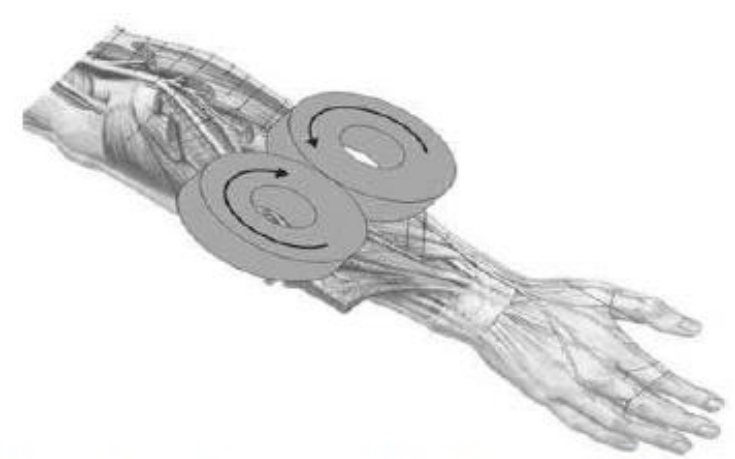

Fig 1. Estimulação magnética de nervo periférico.

Para se determinar se uma configuração de uma bobina, aliada ao circuito elétrico do estimulador, será capaz de gerar estímulos efetivos na região de interesse, são realizadas simulações onde inicialmente se estuda o comportamento do circuito elétrico, em seguida se determina a intensidade do campo elétrico induzido em determinado ponto do tecido nervoso alvo (bem como o gradiente desse campo) e por fim, se analisa através do modelo biofísico se o estímulo gerado irá, ou não, produzir um potencial de ação. [2]- [3]

O uso do método dos Elementos Finitos nesse trabalho visa facilitar o cálculo do gradiente do campo em pontos internos do corpo humano.

\section{O modelo de Hodgkin-Huxley}

O modelo de Hodgkin-Huxley (H-H) descreve o comportamento dinâmico do potencial da membrana celular quando ela é submetida a um estímulo externo. [2]-[3] O modelo H-H é baseado no axônio gigante de lula e é o primeiro modelo iônico que descreve a excitação da membrana.

Hodgkin e Huxley consideraram a membrana da célula como vários ramos paralelos independentes por onde a corrente passa, como mostrado na Fig 2. O modelo determina a variação da condutividade da membrana celular que está alinhada ao longo da direção $\mathrm{x}$ - a certos íons e consegue prever o mecanismo de ativação celular.

Os canais iônicos ativos presentes na célula, denominados de bombas iônicas, por produzirem um gradiente iônico através da membrana, são representados por potenciais, associadas aos canais iônicos de sódio, potássio e outros íons.

Como a membrana está submetida a um gradiente iônico entre suas faces, ela exibe um efeito capacitivo representado pela capacitância por unidade de comprimento. Além disso, também é considerada a resistência longitudinal por unidade de comprimento.

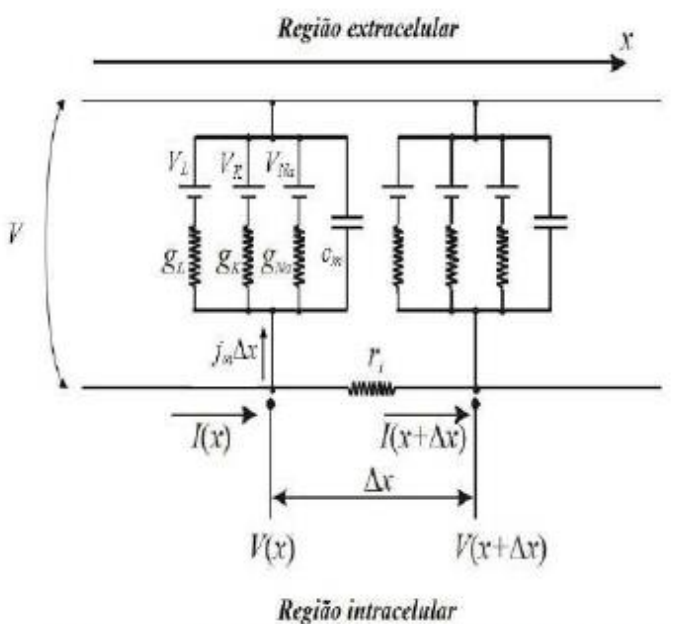

Fig 2. Modelo de parâmetros distribuídos para a membrana de um axônio. 
Após análise e manipulações algébricas, verificou-se que o comportamento dinâmico do potencial transmembrana é determinado pela seguinte equação diferencial:

$$
\Lambda^{2} \frac{\partial^{2} \mathrm{~V}}{\partial \mathrm{x}^{2}}-\mathrm{V}=\frac{\partial \mathrm{V}}{\partial \mathrm{t}}+\Lambda^{2} \frac{\partial \mathrm{E}_{\mathrm{x}}}{\partial \mathrm{x}}
$$

onde os parâmetros $\Lambda$ e t são funções do potencial de membrana.

Observando essa equação, observa-se que a não homogeneidade representada pelo gradiente longitudinal do campo elétrico é capaz de alterar a resposta do sistema desde uma simples alteração difusa até a propagação de um impulso em forma de onda. [2] A esse termo chamamos de função de ativação.

\section{O Circuito Estimulador}

O método mais comumente empregado para se gerar um campo magnético variável é a rápida descarga de um banco de capacitores através de uma bobina. Para isso, utiliza-se um circuito RLC, modelado pela equação diferencial: [1]-[2],[4]-[6]

$$
\frac{d^{2} i}{d t^{2}}+\frac{R}{L} \frac{d i}{d t}+\frac{1}{L C} i=0
$$

O circuito básico utilizado pela TMS capaz de gerar os efeitos descritos é indicado na Fig. 3, e é formado basicamente por um capacitor, um indutor (bobina), um resistor, uma chave semicondutora e um circuito de controle que é responsável pela geração de pulsos e pode ser pré- configurado de acordo com a aplicação.

Uma vez que a estimulação do tecido é provocada pela indução de uma corrente de densidade suficiente, que é proporcional à taxa de variação com o tempo da densidade do fluxo magnético (ou seja, $\mathrm{d} \mathbf{B} / \mathrm{dt}$ ), é importante projetar o circuito de estimulação que permita essa variação. [2]

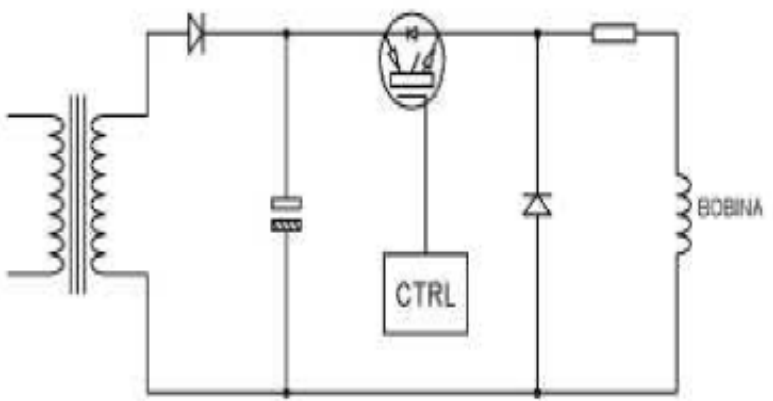

Fig 3. O circuito RLC da TMS.
Esses circuitos estimuladores devem prover uma exata variação da corrente, pois apenas valores específicos de amplitude, duração e sentido serão capazes de estimular determinada estrutura nervosa, a fim de se executar específico tipo de tratamento de neuropatias.

A corrente determinada na Fig. 3 é do tipo monofásica, dado que os parâmetros determinam resposta sobreamortecida. Pode-se ver esse tipo de resposta na Fig. 4.

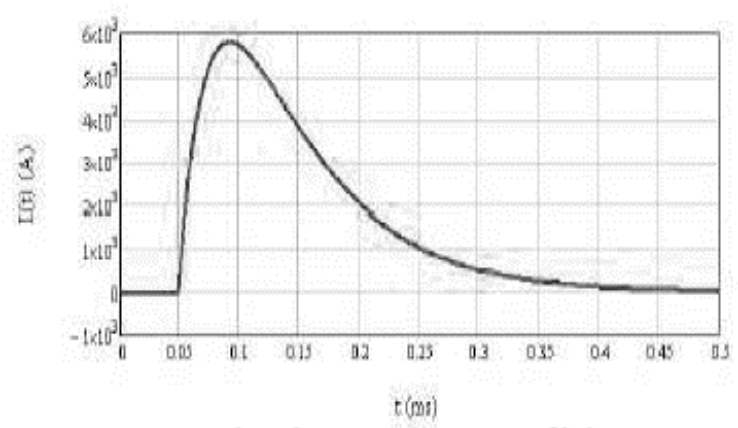

Fig 4. Pulso de corrente moonofásica.

\section{Modelamento matemático da cabeça humana usando o MEF}

Apesar da técnica da estimulação magnética também ser utilizada na estimulação de nervos periféricos, o alvo principal dos pulsos eletromagnéticos é o córtex cerebral, onde os estímulos podem atingir áreas relacionadas a diversas doenças que afetam o sistema nervoso. [1]-[3]

A estimulação desses tecidos por campos magnéticos leva uma grande vantagem sobre a estimulação elétrica, pois o campo magnético quase não sofre atenuações nos tecidos biológicos, enquanto a corrente elétrica precisa atravessar regiões que atuam como isolantes elétricos (ossos, gordura, pele, etc).

O cálculo dos campos no espaço é feito através das equações clássicas da eletrodinâmica, mas no interior dos tecidos (homogêneos ou não), se torna mais difícil.

Já que os tecidos humanos apresentam tanto propriedades eletromagnéticas, como também geometrias complexas, um modelo analítico de tal problema seria extremamente complicado. Em razão disto, deve-se fazer uso de um método numérico ao invés de um método analítico.

O método escolhido foi o método dos elementos finitos (MEF) que apresenta grande eficiência em domínios com condições de contorno complexas. [6]- [9] 
No MEF, um sistema contínuo se transforma em um sistema discreto que consiste em um número finito de elementos cujas geometrias são mais simples. Essas geometrias são ligadas por nós, dessa forma uma estrutura em forma de malha ou 'mesh' é criada para que então a variável desejada seja encontrada. Tais elementos também podem modelar domínios não homogêneos, assumindo que cada elemento é homogêneo. [6]-[9] Na Fig. 5 é mostrada uma discretização de uma esfera multicamadas, cada camada representaria um tecido diferente da cabeça. A equação diferencial parcial pode então ser resolvida aproximadamente através da resolução de um sistema de equações lineares. Quanto maior o número de subdivisões do domínio, maior será a aproximação da resposta exata.

No presente trabalho, usou-se um modelo esférico homogêneo para representar a cabeça humana. Tal modelo está representado na Fig.6.

Então, neste projeto conseguiu-se calcular a distribuição do campo vetorial elétrico no espaço tridimensional a partir da solução da equação de Laplace, ou seja, para os campos eletrostáticos que são os campos invariantes no tempo. Esse problema é regido pela seguinte equação:

$$
\frac{\partial^{2} \mathrm{~V}}{\partial \mathrm{x}^{2}}+\frac{\partial^{2} \mathrm{~V}}{\partial \mathrm{y}^{2}}+\frac{\partial^{2} \mathrm{~V}}{\partial \mathrm{z}^{2}}=0
$$

em que $\mathrm{V}$ é o potencial elétrico no ponto $\mathrm{P}(\mathrm{x} 0, \mathrm{y} 0, \mathrm{z} 0)$.

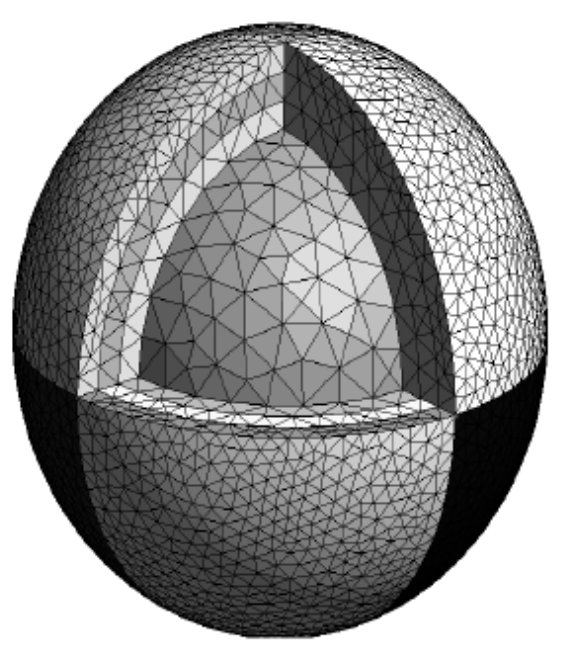

Fig. 5. Esfera heterogênea multicamadas discretizada.

Nas etapas iniciais, estudamos o cálculo do gradiente de temperaturas via MEF em regimes permanentes. Estudou-se primeiramente o caso da condução de calor em planos e depois em volumes cúbicos. Essa metodologia foi adotada, pois a equação de transferência de calor em regime permanente é basicamente a mesma equação que rege a eletrostática.
O software Gmsh versão 2.4.2 foi usado para fazer a discretização do domínio. Nessa discretização, foram utilizados elementos tetraédricos de primeira ordem, obtendo no total 328 nós e 1466 elementos.

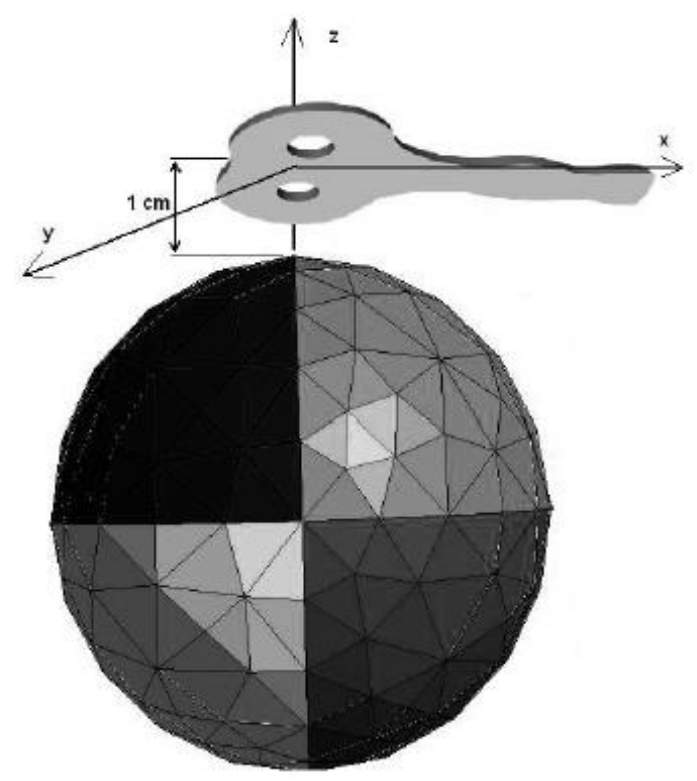

Fig. 6. Esfera homogênea discretizada.

Observando a Fig. 6, percebemos a presença da bobina de estimulação, posicionada no eixo z, que passa pelo centro da esfera. Para essa bobina, foi-se calculado o potencial elétrico em 14 nós através da formulação desenvolvida por [2], enquanto aos outros nós foi-se atribuído o potencial zero. Esses potenciais foram inseridos como condição de contorno de Dirichlet. [5]

Nesta análise, a bobina considerada foi do tipo "butterfly", com $\mathrm{r}=2,3 \mathrm{~cm}$ (raio das espiras da bobina) e $\mathrm{a}=180^{\circ}$ (ângulo entre as espiras) em $\mathrm{t}=0 \mathrm{~s}, \mathrm{~N}=10$ (número de voltas em cada espira).

A cabeça foi modelada como uma esfera homogênea de raio de $10 \mathrm{~cm}$. Os parâmetros do circuito estimulador encontram-se na tabela 1 :

\section{Tabela 1. Valores dos elementos do circuito usado}

\begin{tabular}{|c|c|}
\hline elemento & Valor \\
\hline Capacitor & $200 \mu \mathrm{F}$ \\
\hline Indutor & $10 \mu \mathrm{H}$ \\
\hline Resistência & $0,5 \Omega$ \\
\hline
\end{tabular}

A tensão inicial do banco capacitores é de $4000 \mathrm{~V}$. 
Então, os valores da função de ativação $\partial \mathrm{Ex} / \partial \mathrm{x}$ em alguns pontos foram calculados pelo método descrito em [2], tais pontos, que foram utilizados como condição de contorno Dirichlet, estão indicados na tabela 2 abaixo:

Tabela 2. Condições de contorno

\begin{tabular}{|c|c|c|}
\hline Ponto & $\begin{array}{c}\text { Coordenanada } \\
(\mathrm{cm})\end{array}$ & $\begin{array}{c}\partial \mathrm{E}_{\mathrm{x}} / \partial \mathrm{x} \\
\left(\mathrm{mV} / \mathrm{cm}^{2}\right)\end{array}$ \\
\hline A & $(0,0,-1)$ & 0 \\
\hline B & $(5,5,-3.93)$ & 1.217 \\
\hline C & $(5,-5,-3.93)$ & -1.217 \\
\hline D & $(-5,5,-3.93)$ & -1.217 \\
\hline E & $(-5,-5,-3.93)$ & 1.217 \\
\hline F & $(7.07,7.07,-11)$ & 0.116 \\
\hline G & $(7.07,-7.07,-11)$ & -0.116 \\
\hline H & $(-7.07,7.07,-11)$ & -0.116 \\
\hline I & $(-7.07,-7.07,-11)$ & 0.116 \\
\hline J & $(5,5,-18.07)$ & 0.015 \\
\hline K & $(5,-5,-18.07)$ & -0.015 \\
\hline L & $(-5,5,-18.07)$ & -0.015 \\
\hline M & $(-5,-5,-18.07)$ & 0.015 \\
\hline N & $(0,0,-21)$ & 0 \\
\hline
\end{tabular}

Nos demais pontos a variável foi encontrada via MEF, através de algoritmo desenvolvido em MATLAB. Também foi desenvolvido algoritmo em $\mathrm{C}++$, devido à sua maior velocidade de computação.

A Fig. 7 mostra os resultados obtidos no plano xz definido pela Fig. 6.

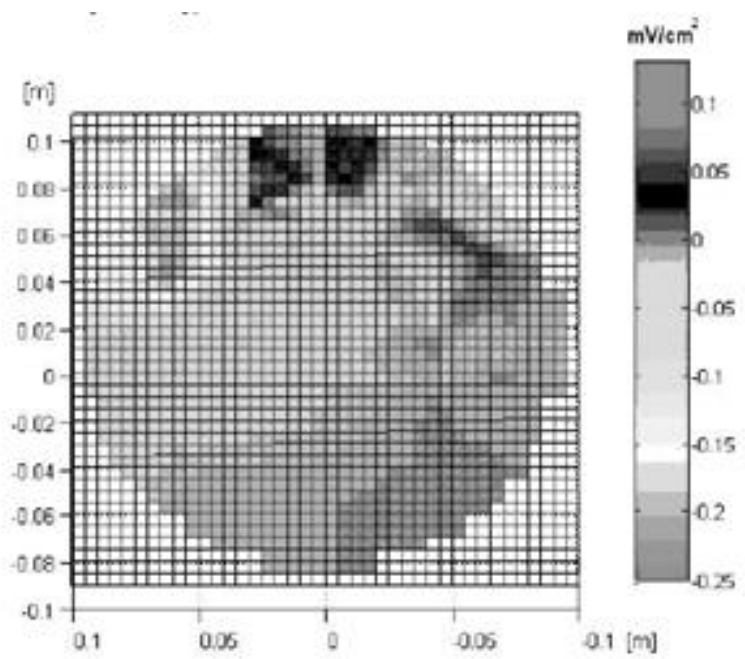

Fig. 7. Gradiente do campo elétrico induzido para $y=0$

\section{Discussões}

No âmbito do desenvolvimento deste trabalho, foram geradas contribuições para o estudo da estimulação magnética através de uma abordagem via MEF. Inicialmente desenvolveu-se um algoritmo robusto escrito em $\mathrm{C}++\mathrm{e}$ em MATLAB através do formalismo matemático exigido pelo MEF em que, com simulações que convergem a um resultado, pode-se determinar a distribuição do gradiente do campo elétrico induzido na cabeça humana através de uma formulação baseada na eletrostática.

A entrada de dados é feita através de duas matrizes definidas em dois arquivos de texto previamente geradas por um software discretizador de domínios chamado gmsh. O processo computacional necessário ao cálculo da distribuição do gradiente do campo elétrico induzido, que utiliza processo analítico, [2],[3] é reduzido ao cálculo em alguns pontos e, após modelamento via MEF, encontra-se o valor da variável nos demais pontos.

\section{Referências}

[1] E. M. Wassermann, C. M. Epstein, Oxford Handbook of Transcranial Stimulation, Oxford University Press, 2008.

[2] FEITOSA, M. A. F. Contribuições para o projeto de estimuladores magnéticos. 2009. 103 f. Tese (Doutorado em Engenharia Elétrica) - Centro de Tecnologia e Geociências - Universidade Federal de Pernambuco, Pernambuco. 2009.

[3] FEITOSA, M. A. F.; FONTANA, E., "Solução série para o campo elétrico induzido por estimuladores magnéticos na geometria slinky generalizada", Anais do XVI Congresso Brasileiro de Automática, Salvador, Sociedade Brasileira de Automática, 2006, pp. 3391-3396.

[4] Cai D., Fang Y., Cao X., "Transcranial magnetic stimulation: modeling, calculating and system design”, 2nd International Conference on Digital Object Identifier, 2009, pp. 1-5.

[5] V. T. Kastreva, S. P. Papazov, e I. K. Daskalov, "Peripheral nerve magnetic stimulation: influence of tissue non-homogeneity", BioMed Central, 2003.

[6] S. Yang., G. Xu., Y. Chen., et al, "3D Realistic Head Model Simulation Based on Transcranial Magnetic Stimulation", Engineering in Medicine and Biology Society, 2006, pp. 6469-6472. 
[7] O. C. Zienkiewikz, The Finite Element Method, Oxford: Butterworth-Heinermann, 2000.

[8] Matthew N. O. Sadiku, Numerical Techniques in Electromagnetics, $3^{\mathrm{a}} \mathrm{ed}$. CRC Press, 2009.

[9] Jianming Jin, The Finite Element Method in Eletromagnetics, John Xiley \& Sons, 1962. 